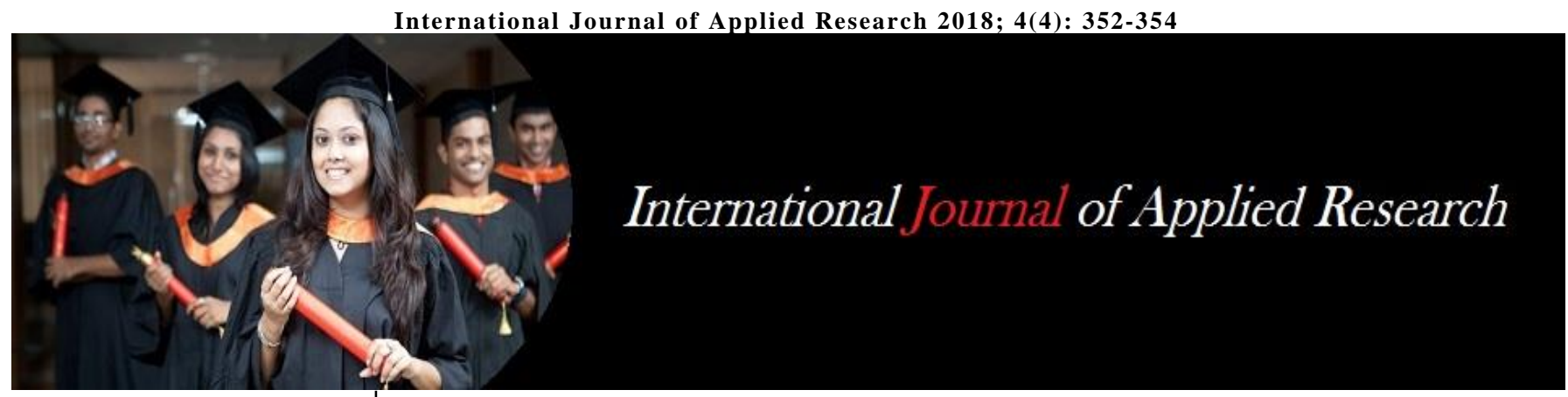

ISSN Print: 2394-7500 ISSN Online: 2394-5869 Impact Factor: 5.2

IJAR 2018; 4(4): 352-354 www.allresearchjournal.com

Received: 24-02-2018 Accepted: 29-03-2018

Anand Mohan

J.R.F (DST), Government of India, P.G Department of Physics C.M. Science College, Darbhanga, Bihar, India
Corresponding Author: Anand Mohan

J.R.F (DST), Government of India, P.G Department of Physics C.M. Science College, Darbhanga, Bihar, India

\title{
Optical-antenna: Great attraction for communication community
}

\section{Anand Mohan}

DOI: https://doi.org/10.22271/allresearch.2018.v4.i4e.7505

\section{Abstract}

The Dielectric Resonator Antenna (DRA) has mainly focus on good quality communication with low profile Antenna. Cylindrical Dielectric Resonator optical Antenna (CDROA) is compact in structure, light in weight conformable to surface planar some natural carbon atom form hollow cylinder without side diameter of only one nano meter. The Resonator Antenna is well suited for microwave devices integration and feeding Technique and, especially with the microwave integrated circuit technology. This unique type antenna has unique applications for direct broadcast Technique as well as satellite system on satellite communication at global positioning system (GPS) and high frequency navigation system and good accuracy and a large variety of radar systems demand for good quality Antenna for the best performance of antenna system.

Keywords: Antenna resonator, nano meter, ICT network, Dielectric Resonator box on bandwidth

\section{Introduction}

The ICT network communication is developing very rapidly with passage of time. The high speed mobile approaches to new technologies are being introduced to facilitate the mobile users from the technology ${ }^{[1-15]}$. The Dielectric Resonator Antenna has unique type of the antenna which can exhibit various advantages over conducting surface of antenna. The evolution in the field of microwave communication in the last two or three decades, especially that of the internet and the shrinking size of communicational devices likes mobile phones to pocketsize smart phones/Android phone, have transformed the world into a global village CDRA has able to transformation and reform the size of the cylindrical dielectric resonator antenna is defined accordingly with its aspect ratio. The communication system that is needed some more specific and additional features added to the antenna to compensate for the deficiencies and encountered in system for best performance ${ }^{[16-20]}$. There are many method by which DRA have been designed by many researchers for various applications like optical DRA for Mobile Ad-hoc network \& feeding technique although the optical-DRA antennas are capable enough to fulfill all the operational requirements, the temperature conditions are constrained to face certain limitations to avoid these constraints performance of optical Dielectric Resonator Antennas (ODRAs) is evlaluated by their new applications are proposed ${ }^{[21-22]}$. The antenna as per the requirement of various applications so it is necessary to find the control over requirement by the transmitting or receiving device or the locality where it have to be installed. The optical antenna uses different feeding Technique for their excitation such as probe feed; lumped port feed, excitation by different fields and many more techniques are uses by which an antenna system for excitation ${ }^{[23-24]}$. The robust wireless networks that delivers the performance necessary to support emerging application for body Area Network (WLAN) currently supplement or replace and achieving tenability for optical frequencies although wire material operation conduction band interactions among (CDOA) When we increase the dimension of the antenna in direction normal to ground plane, it improves the gain or directivity. Effect of the dielectric constants of the Dielectric Resonator box on bandwidth of the antenna as well as the effect of thickness of the substrate of the device. Optical dielectric Resonator Antenna can be changed by many methods of the variation in the position of bands in antenna as well as gain some of these are studied to find the control over bandwidth and gain of optical antenna radiation patterns also uses many types of antenna used for many purpose of communication, which is able to achieved

$$
\sim 352 \sim
$$


smartly to improves it's of communication system which is depend upon various parameters. The Current trends of information communication technology works on concept of architecture, which is dependent part of complex, which has proposed in this research article ${ }^{[25]}$. Nanoantennas are small in size in conventional antenna which works or operate at the optical frequencies. Optical DRA antenna has high permittivity with low reduction optical DRA allowing numerous application DRA Proportional permittivity in material. The Antenna field structures do not need $\lambda$ bandwidth in Advance generation communication system have very high sensitivity material for digital community with high efficiency. The Antenna not only transmitting and receiving devices it is key part. The dielectric resonator Antenna has good networking to connect one device to another device.

\section{Optical dielectric resonators antenna design at THZ scale frequencies}

Optical antenna has ability to transmit optical frequency with nanoscale wavelength. Antenna provides high gain permittivity of antenna material has great potential to achived Radiation in db-deviation is increased with small radiation variation pattern of Antenna wavelength. Simple we can say that transmitting and receiving equipment (system) is called Antenna. The optical antenna concept has great approach to propagate Antenna system. We have proposed unique type dielectric resonator optical antenna which works on nanometer scale. Optical antenna has unique consideration to optimize energy as well as radiation efficiency may vary optimal. Generally traditional antenna uses dielectric material to make its structure to design by high level software may be possible to manipulate or, simulation based demonstration for dielectric Antenna. In this work we have applied optical DRA antenna to control multichannel receiver with high band width for optical communication. This approach should work well with Dielectric Optical Antenna. Limitations which consider to enhance the spontaneous emission is favourable to obtain exact wavelength for minimizing the gap \& set smaller range gap for unique manufacture technique with high permittivity and emission has enhanced and perfect condition performance at $700 \mathrm{~nm}$ scale boundary.

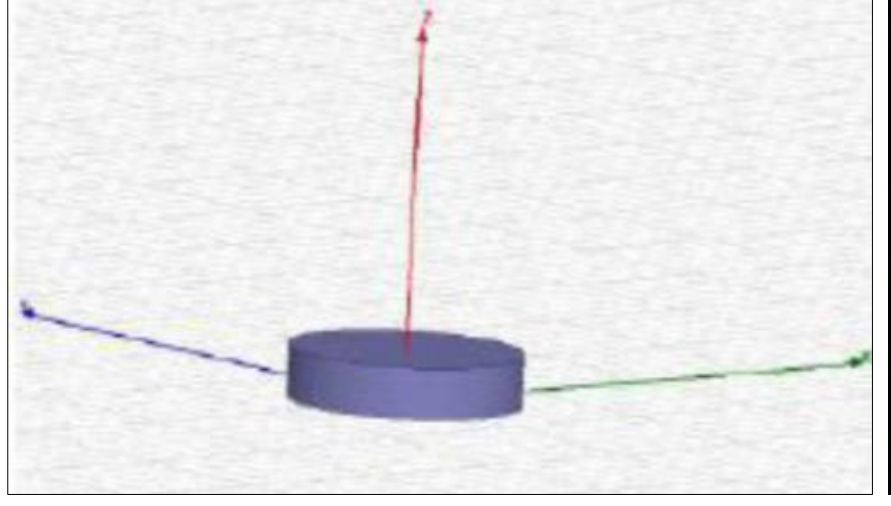

Fig (a): Optical antenna Wavelength scale

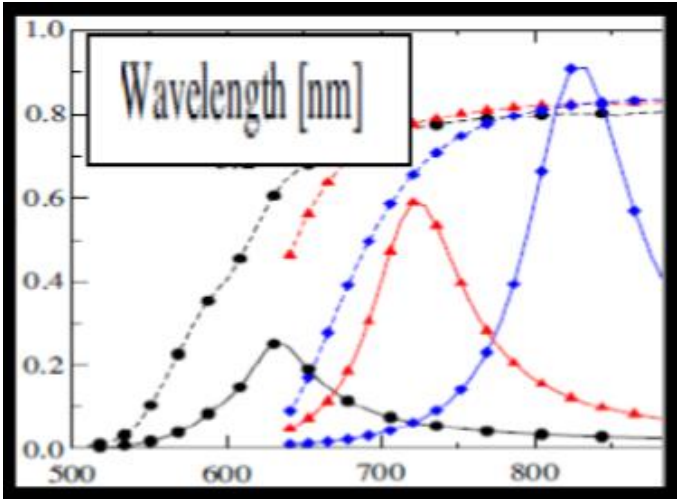

Fig (b): wavelength for minimizing at $\mathrm{nm}$

\section{Conclusions}

In this paper we have studied as well as proposed new method to design optical Antenna which has enable to study this radiation distribution of electromagnetic range or nm range. This research work will very helpful to improve Antenna efficiency at nanoscale. In this Research paper is totally concentrated all about Antenna material, Application, Wavelength scale, fabrication methodology which has high band width for optical communication system to integrate Antenna design for maxima level transmitting efficiency. We have simulated new approach work for Dielectric Antenna. We have presented very new tread communication Arial at nanoscales operation with high bandwidth application to nonmaterial Antenna design due to its high impedance at $\mathrm{nm}$ frequency range. Optical Antenna (Arial) promotes to utilise Antenna materials at terahertz and optical range. We have used the Gold particle. Which has high efficiency for Antenna geometry. We have choosed wavelength at $700 \mathrm{~nm}$ scale. The optical properties of Antenna material has important factor to design Antenna at $\mathrm{nm}$ scale. In this paper we have proposed unique type Dielectric optical Antenna which has special feature for communication networks to be analyzed. The frequency response depends upon optical response of Gold-particle. Optical Antenna has very unique ability to transmit optical frequencies at nanoscale wavelength.

\section{References}

1. Mohan A. The advanced generation mobile broadband technology for wireless communication system and its applications. IJAR 2015;1(12):383-385.

2. Mohan Anand. Radiation Characteristic of Metallic Nano- Particle with Application to Nano-Antenna. An Experimental Study of Effect of Amalkirasayan and Amalkiswaras with Help of Electron Microscopy 2014;5(6):45.

3. Mohan Anand. Synthesis of bi-metallic nanoparticles and analysis of their performances. An experimental study of effect of amalkirasayan and amalkiswaras with help of electron microscopy 2014;5(6):59.

4. Mohan A. Study of Plasmonic Nano Antennas and Their Optimization., International Journal of Emerging Research in Management \&Technology ISSN: 22789359 (Volume-5, Issue-5) Special Issue on International Conference on Advances in Engineering (ICAE) -2016 Conference Held at Hotel Magaji Orchid, Sheshadripuram, Bengaluru, India, 2016.

5. Mohan Anand. Role of Nanoantenna System In Transforming Thermal Energy, Airo International Research Journal ISSN: 2320-3714, 2016, 7.

6. Mohan Anand. IOT: A Big Revolution for Nanoscience, $7^{\text {th }}$ virtual Nanotechnology Poster Conference. 
http://www.nanopaprika.eu/group/nanoposter/page/p1721

7. Sachchida Nand Singh, Ashok Kumar, Anand Mohan. Study of nanoantennas for enhanced Optical emission, Proceedings of International Conference on Advances in Light Technologies and Spectroscopy of Materials (ICALTSM, 2016, 256.

8. Akyildiz IF, Jornet JM, Han C. Terahertz band: Next frontier for wireless communications. Physics Communications 2014;12:16-32. DOI:10.1016/j.phycom. 2014.01.006

9. Anand Mohan. Cylindrical dielectric resonator antennas (CDRA) \& its applications for human life, ISCA, Souvenir of $4^{\text {th }}$ International Virtual Congress IVC2017, ISBN: 978-93-84659-68-4, 2017.

10. Nagatsuma T, Ducournau G, Renaud CC. Advances in terahertz communications accelerated by photonics. Nature Photonics 2016;10(6):371-379.

11. Anand Mohan, Ashok Kumar. Uses of Optical Nanoantenna in ICT and its Ability, Souvenir of $3^{\text {rd }}$ International Virtual Congress, $3^{\text {rd }}$ International Virtual Congress IVC, 2016. ISBN: ISBN: 978-93-84648-78-7

12. Anand Mohan. Study of Plasmonic Nano-Materials for Surface enhanced Localized Surface Plasmon Resonance Spectroscopy (LSPR) \&Their Applications for Optical Antennas. Indian Journal of Agriculture and Allied Sciences, ISSN 2395-1109 2015;1:4.

13. Scholl JA, García-Etxarri A, Koh AL, Dionne J. A. Observation of Quantum Tunneling between Two Plasmonic Nanoparticles. Nano Lett. 2013;13:564-569.

14. Farahani JN, Pohl DW, Eisler H.-J, Hecht B. Single quantum dot coupled to a scanning opticalantenna: A tunable superemitter, Phys. Rev. Lett 2005;95(1):017402.

15. Kühn S, Håkanson U, Rogobete L, Sandoghdar V. Enhancement of single-molecule Fluorescence using a gold nanoparticle as an optical nanoantenna, Phys. Rev. Lett 2006;97(1):017402.

16. Muskens OL, Giannini V, Sanchez-Gil JA, Gómez Rivas J. Strong enhancement of the radiative decay rate of emitters by single plasmonic nanoantennas, Nano Lett. 2007; 7(9):2871-2875.

17. Bakker RM, Yuan H.-K, Liu Z, Drachev VP, Kildishev AV, Shalaev VM et al. Enhanced localized fluorescence in plasmonic nanoantennae, Appl. Phys. Lett 2008;92(4):043101.

18. Patel SK, Argyropoulos C. Plasmonic nanoantennas: enhancing light-matter interactions at the nanoscale. EPJ Appl. Metamat. 2015; 2:4.

19. Anger P, Bharadwaj P, Novotny L. Enhancement and quenching of single molecule fluorescence. Physical Review Letters 2006;96(11):113002.

20. Bharadwaj P, Novotny L. Spectral dependence of single molecule fluorescence enhancement. Optical Express 2007;15(21):14266-14274.

21. Hartschuh A, Qian H, Meixner AJ, Anderson N, Novotny L. Nanoscale optical imaging of excitons in single-walled carbon nanotubes. Nano Letters 2005;5(11):2310.

22. Novotny L, Hecht B. Principles of Nano-Optics. Cambridge: Cambridge University Press, 2006.

23. Petosa A, Ittipiboon A. Dielectric resonator antennas: A historical review and the current state of the art," IEEE Antennas and Propag Mag 2010, 52.
24. Nagatsuma T, Ducournau G, Renaud CC. Advances in terahertz communications accelerated by photonics. Nature Photonics 2016;10(6):371-379

25. Xu Q, Pan C, Chen Y. Nano-optical antenna sensor based on localized surface plasmon resonance. IEEE International Conference on High Voltage Engineering and Application (ICHVE), Chengdu 2016, 1-4. doi: 10.1109/ICHVE.2016.7800683 